\title{
Bidirectional ocular dominance plasticity of inhibitory networks: recent advances and unresolved questions
}

\section{Gordon B. Smith and Mark F. Bear*}

Department of Brain and Cognitive Sciences, Howard Hughes Medical Institute, The Picower Institute for Learning and Memory, Massachusetts Institute of Technology, Cambridge, MA, USA

Edited by:

Barry W. Connors, Brown University, USA

\section{Reviewed by:}

Jay Gibson, The University of Texas Southwestern Medical Center, USA Barry W. Connors, Brown University, USA

\section{*Correspondence:}

Mark F. Bear, Howard Hughes Medical Institute, The Picower Institute for Learning and Memory, Department of Brain and Cognitive Sciences,

Massachusetts Institute of Technology, Cambridge, MA 02139, USA.

e-mail: mbear@mit.edu
Monocular visual deprivation (MD) produces profound changes in the ocular dominance (OD) of neurons in the visual cortex. MD shifts visually evoked responses away from the deprived eye and toward domination by the open-eye. Over 30 years ago, two different theories were proposed to account for these changes: either through effects on excitatory visual drive, thereby shifting the balance of excitation in favor of the open-eye, or through effects on intracortical inhibition, thereby suppressing responses from the deprived eye. In the intervening years, a scientific consensus emerged that the major functional effects of MD result from plasticity at excitatory connections in the visual cortex. A recent study by Yazaki-Sugiyama et al. (2009) in mouse visual cortex appears to re-open the debate. Here we take a critical look at these intriguing new data in the context of other recent findings in rodent visual cortex.

Keywords: ocular dominance plasticity, monocular deprivation, inhibition, visual cortex

\section{A BRIEF HISTORY: INHIBITION, OCULAR DOMINANCE, AND OCULAR DOMINANCE PLASTICITY}

Ocular dominance (OD) plasticity is the preeminent model of experience-dependent plasticity in the mammalian nervous system. Retinotopically matched inputs from both eyes converge on individual cortical neurons to produce binocular vision. The strength and persistence of these connections is highly susceptible to manipulations that asymmetrically degrade the quality of visual experience, such as monocular deprivation (MD, Wiesel and Hubel, 1963). Awake recordings in juvenile mice have shown that a brief period of monocular lid suture produces an OD shift solely through the loss of responsiveness to the deprived eye (Frenkel and Bear, 2004). Broadly speaking, there are two categories of changes that could account for the observed reduction in deprived-eye responses, which are not mutually exclusive. First, excitatory drive into the cortex from the deprived eye could become weakened, thereby decreasing responses driven by the deprived eye. Second, intracortical inhibition of deprived-eye inputs could increase following MD, thereby suppressing or "masking" visual responses evoked by the deprived eye.

A large body of classic work in kittens has supported the former idea, demonstrating that MD leads to a reduction in cortical innervation by excitatory thalamocortical axons subserving the deprived eye (Shatz and Stryker, 1978), which is accompanied physiologically by considerable weakening of deprived-eye inputs (Singer, 1977; Tsumoto and Suda, 1978; Mitzdorf and Singer, 1980). The alternative hypothesis, that the OD shift observed following MD results from altered intracortical inhibition, was originally suggested over 30 years ago, again based on experiments in kittens (Duffy et al., 1976; Burchfiel and Duffy, 1981). Local iontophoretic administration of bicuculline, a $\mathrm{GABA}_{\mathrm{A}}$ receptor antagonist, restores binocularity and deprived-eye responses in cortical neurons following $\mathrm{MD}$ (Burchfiel and Duffy, 1981). However, attributing these results to MD-induced plasticity of inhibitory networks is difficult, as blockade of GABA receptors in normal cats both broadens receptive field properties and unmasks previously subthreshold responses (Sillito, 1975). A similar unmasking was also observed for responses evoked through either the deprived or non-deprived eye in MD animals (Sillito et al., 1981).

In recent years rodents have emerged as the preferred system for studies of visual cortical plasticity, and questions of the relative contribution of MD-induced changes in excitation and inhibition have been readdressed with new approaches. Several lines of evidence demonstrate that the mechanisms of long-term depression (LTD) of excitatory synapses play a central role in the weakening of visual responses (reviewed in Smith et al., 2009). Brief MD leads to reduced surface expression of AMPA-type glutamate receptors and occludes subsequent induction of LTD (Heynen et al., 2003; Crozier et al., 2007). Furthermore, molecular manipulations that block AMPA receptor endocytosis exclusively in excitatory neurons completely prevent the loss of deprived-eye responses following MD (Yoon et al., 2009; McCurry et al., 2010). In addition, it has recently been shown that the full OD shift in cortical layer 4 is expressed by modifications of excitatory thalamocortical synaptic currents (Khibnik et al., 2010).

However, additional work has shown that cortical inhibitory networks can also be strongly affected by MD. Brief periods of deprivation induce potentiation of feedback inhibition involving fast-spiking (FS) basket cells in layer 4 of the monocular visual cortex in rats studied ex vivo (Maffei et al., 2006). Additionally, two recent studies used calcium imaging in mice in vivo and found MD-induced OD shifts in both excitatory and inhibitory neurons (Gandhi et al., 2008; Kameyama et al., 2010). Thus, both the inputs to and outputs from inhibitory neurons can be modified by deprivation. 
In a recently published report in the journal Nature, YazakiSugiyama et al. (2009) take a direct approach to study plasticity of inhibitory interneurons and describe a novel and surprising response of these cells to MD. The authors used technically demanding in vivo intracellular recordings in anesthetized mice to assay the OD of individual cortical neurons. This approach enabled them to directly compare OD and the consequences of $\mathrm{MD}$ in pyramidal neurons and FS interneurons. In addition, by slowly infusing picrotoxin (PTX) into pyramidal neurons, they could examine the contribution of somatic inhibition to OD and the expression of OD plasticity. This approach enabled the investigators to address a number of interesting questions, discussed below.

The aims and approaches used in the study by Yazaki-Sugiyama are truly laudable. However, ambitious experiments often impose technical limitations that can make interpretation difficult. As discussed below, some of their findings are not easily reconciled with other recent results. Thus, the conclusions reached by YazakiSugiyama, while interesting and provocative, will require confirmation using additional approaches.

\section{HOW DOES OD DIFFER IN EXCITATORY AND INHIBITORY CELLS, AND HOW IS IT SHAPED BY INHIBITION?}

In pyramidal neurons recorded from control (non-MD) mice, visually evoked spiking displays a biased response, with greater firing elicited by stimulation of the contralateral eye. The degree of contralateral bias observed with intracellular recordings (roughly 2:1) is similar to what has been observed previously using either extracellular single-unit recordings or visually evoked potentials (Gordon and Stryker, 1996; Frenkel and Bear, 2004), and matches the relative anatomical density of projection neurons in the LGN (Coleman et al., 2009). Interestingly, however, intracellular recordings from FS interneurons, putatively parvalbumin-positive large basket cells, exhibited strongly binocular responses, without bias toward either eye (Yazaki-Sugiyama et al., 2009) (Figure 1, left column).

The observed binocularity of FS cells is consistent with prior results from suspected interneurons recorded in awake rabbits (Swadlow, 1988), however these results differ from two recent studies using in vivo calcium imaging in mice, which report strong contralateral biases in genetically labeled inhibitory cells. In GAD67GFP knock-in mice, both excitatory and inhibitory neurons display similar OD distributions with contralateral biases (Gandhi et al., 2008). Similarly, inhibitory cells in VGAT-Venus transgenic mice, which although more binocular than excitatory neurons, still on average exhibit a strong contralateral bias (Kameyama et al., 2010). Additionally, in parvalbumin-positive interneurons from normally reared rats, visually evoked c-Fos induction shows a 2:1 contralateral bias (Mainardi et al., 2009).

The basis for the different results reported in these studies and by Yazaki-Sugiyama et al. remains to be determined. Although GAD67GFP mice have reduced cortical GABA levels which may impact the normal development of OD (Tamamaki et al., 2003; Hensch, 2005), this is not the case in VGAT-Venus mice (Kameyama et al., 2010). An additional explanation proposed by Yazaki-Sugiyama et al. is that the results of in vivo calcium imaging are misleading because these experiments are restricted to superficial layers of the cortex which contain fewer FS cells. However, given that all neurons recorded by Yazaki-Sugiyama et al. were also located in layer 2/3, this is unlikely to account for the different results.

To test the functional consequences of somatic inhibition on OD of excitatory neurons, Yazaki-Sugiyama et al. performed technically challenging recordings from pyramidal neurons in which the $\mathrm{GABA}_{\mathrm{A}}$ antagonist PTX was slowly infused intracellularly through the recording pipette. This technique nominally allows the examination of OD in the same neuron both prior to and following intracellular blockade of $\mathrm{GABA}_{\mathrm{A}}$ receptors, thereby isolating the response to excitatory inputs. In non-MD control mice, PTX greatly increased the binocularity of visually driven responses (Figure 1, left column). Notably, binocularity was increased in both neurons with an initial contralateral bias (the majority of cells) and those with an initial ipsilateral bias.

This surprising result suggests that excitation onto pyramidal cells is binocularly balanced, with the maintenance of OD biases dependent on asymmetric inhibition. This interpretation requires the existence of biased inhibitory networks, which might be expected to include FS cells due to their apparently central role in OD (Hensch, 2005; Katagiri et al., 2007). The finding that FS cells lack such a bias and are in fact strikingly binocular (YazakiSugiyama et al., 2009) suggests that monocularly biased inhibition relies on other interneuron subtypes and that these subtypes play a greater role in modulating pyramidal cell responses than previously thought. Although more complex cortical circuits involving disinhibition could play a role in the observed results, such models also require the existence of monocularly biased inhibition.

The findings are surprising because feed-forward (excitatory) projections from the LGN (targeting layer 4 and deep layer 2/3 in mice) show an anatomical bias favoring the contralateral eye by approximately a 2:1 ratio, equivalent to the physiological response ratio recorded in layer 4 (Frenkel and Bear, 2004; Coleman et al., 2009). Thus, it has been assumed that the contralateral bias of cortical responses simply reflects excitatory innervation density, an idea well supported by recordings from species with anatomically defined OD columns, such as the cat and monkey. The model of balanced OD in excitatory neurons of mouse visual cortex requires new assumptions about how inputs from the two eyes target excitatory and inhibitory neurons, and how these cells interconnect.

The possibility needs to be considered that the apparent loss of biased responses in pyramidal cells following PTX infusion is caused by saturation of pyramidal cell firing rates, which might clip the dominant contralateral eye response and obscure eye-specific differences in excitatory input. Previous results have shown elevated spontaneous firing rates using this technique (Nelson et al., 1994), and there is a trend toward elevated firing rates following PTX in the current study (though not statistically significant). Perhaps this concern could be addressed in future studies by varying the contrast of the visual stimulus or by passing hyperpolarizing current into the neuron before and after PTX infusion.

\section{HOW DOES BRIEF (3-DAY) MD SHIFT OD IN EXCITATORY AND INHIBITORY CELLS, AND HOW IS EXPRESSION OF THE SHIFT SHAPED BY INHIBITION?}

Three days of contralateral eye MD produces a shift in the OD of visual cortical neurons, away from the previously dominant contralateral eye and toward the ipsilateral non-deprived eye (Gordon 
A

\section{Ocular Dominance}

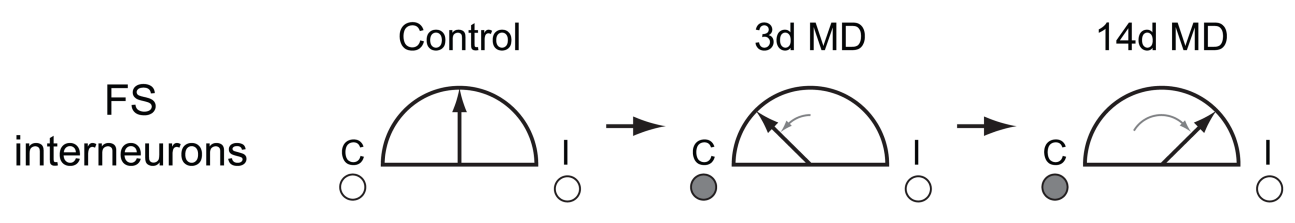

\section{Pyramidal neurons}
Pyramidal neurons + PTX
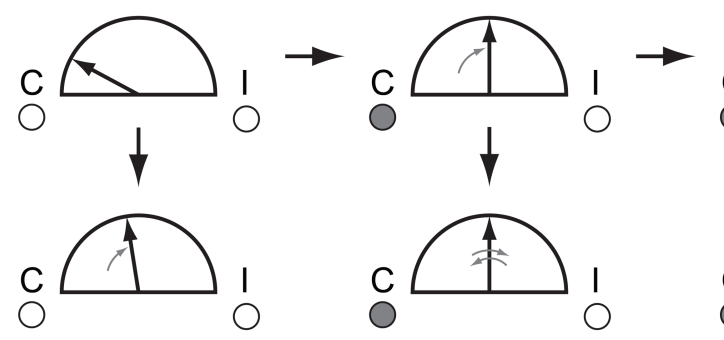

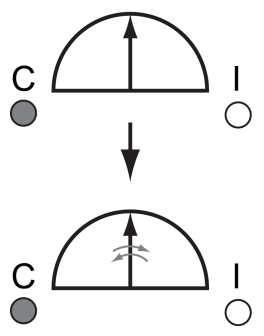

B

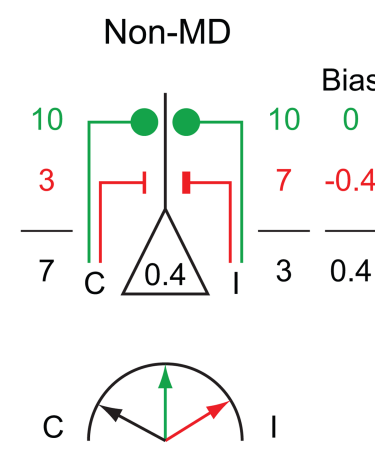

FIGURE 1 | Consequences of bidirectional OD plasticity in fast-spiking GABA circuits. (A) Schematic summary of OD data for FS and pyramidal neurons reported by Yazaki-Sugiyama et al. Black arrows indicate OD bias under a given condition, ranging from purely contralateral-eye responses to purely ipsilateral-eye responses. Small gray arrows indicate changes relative to control (non-MD or no PTX conditions). Top row. In non-MD mice, FS cells exhibit binocular responses, which first shift toward the deprived eye after 3 days MD, then become biased to the open-eye after 14 days MD. Middle row. Pyramidal neurons in non-MD mice display contralaterally biased responses, which become binocular following either 3 or 14 days MD. Bottom row. Spike bias in pyramidal neurons after intracellular PTX application. In non-MD mice, responses become binocular with intracellular PTX, whereas after 3 days MD PTX leads to inversion of OD biases. (B) Schematic representation of the relative strengths of excitatory
Brief (3d) MD

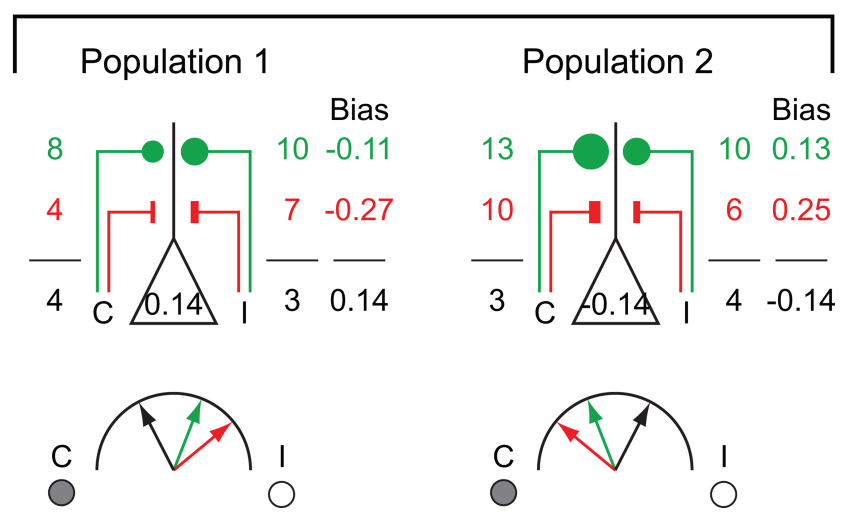

and inhibitory inputs which could potentially produce the observed data. Numbers represent relative excitatory (green) and inhibitory (red) synaptic strengths of inputs driven by stimulation of the contralateral or ipsilateral eye. Number in center of pyramidal neuron is the overall spike bias, and numbers to left and right represent the influences of contralateral and ipsilateral visual stimulation, respectively. Excitatory and inhibitory inputs sum to generate the overall response and resulting spike bias. Left panel: In order to produce contralaterally biased responses in neurons receiving binocularly balanced excitation, ipsilaterally dominated inhibitory inputs (and therefore ipsilaterally dominated inhibitory neurons) are required. Right panels: After MD excitatory neurons are on average binocular, comprised of two populations showing a modest bias to either eye. These overall biases invert in the absence of inhibition, thus excitatory inputs have the opposite OD bias from the neuron itself. and Stryker, 1996; Frenkel and Bear, 2004). In measurements of spike bias from pyramidal neurons following MD, Yazaki-Sugiyama et al. found a similar loss of contralateral bias, with the average response across neurons having become highly binocular. In contrast FS cells, which had binocular responses in control mice, were reported to acquire biased responses and shift toward the deprived (contralateral) eye following MD (Figure 1, middle column).
On the surface, this observation in FS cells again disagrees with Gandhi et al. (2008) who find that the OD of inhibitory neurons in the GAD67-GFP mice does not change from baseline after 3 days of MD. However, recall that the inhibitory neurons studied by Gandhi et al. in non-deprived mice were, like the excitatory neurons, initially biased toward the contralateral eye. Thus, both studies are actually in agreement that inhibitory neurons show a 
contralateral-eye bias after 3 days of MD. The question is whether this reflects a shift in OD, or is rather the maintenance of the baseline, non-deprived state.

An interesting clue to resolving the difference is the finding by Yazaki-Sugiyama et al. that spontaneous activity in FS neurons is dramatically $(>5 \times)$ reduced by $M D$. It is possible that the high spontaneous firing rates in non-MD animals obscure visually evoked responses and could potentially mask an underlying contralateraleye bias in the inputs to FS cells. A reduction in spontaneous activity with MD would then unmask biased responses, leading to a shift in spike output toward the deprived eye. If the spontaneous firing rate of FS cells is in fact a critical determinant of their OD, then the varying reports of biased responses in interneurons may be due to differences in spontaneous firing rates across mouse lines and experimental preparations.

The question of the response to brief MD in inhibitory cells is complicated by the finding that in VGAT-Venus transgenic mice, inhibitory neurons lose their initial contralateral bias and become increasingly binocular following 2 days of MD (Kameyama et al., 2010). This finding appears to disagree with the results of both Yazaki-Sugiyama et al. and Gandhi et al., who report contralaterally biased responses following brief deprivation. As discussed previously, unlike the GAD67-GFP mice studied by Gandhi et al., cortical GABA levels are normal in VGAT-Venus animals, which may partly explain the contrasting results. Additionally, differences in aesthesia and recording technique (intracellular electrodes vs. calcium imaging) may also contribute to the variety of results regarding the response of inhibitory cells to short (2- to 3-day) periods of MD.

Yazaki-Sugiyama et al. used intracellular PTX to block somatic inhibitory inputs and isolate excitatory drive onto pyramidal cells following brief MD (Figure 1, middle column). Infusion of PTX did not change the overall degree of binocularity, suggesting that on a population level, changes in inhibition make a minor contribution to expression of the OD shift. Interestingly, however, PTX infusion produced an inversion of OD for each individual neuron: those exhibiting a contralateral bias prior to $\mathrm{GABA}_{\mathrm{A}}$ blockade became dominated by the ipsilateral eye following intracellular PTX application, and vice versa.

These results suggest that monocularly biased excitation, which was not observed in non-MD control neurons with PTX, emerges following visual deprivation. In addition, the conversion of openeye dominated responses to deprived-eye dominated responses following PTX suggests the presence of inhibitory networks biased to the open-eye after 3 days of MD. Given that open-eye dominated FS cells were not observed after short-term MD, these results again require a key role for other inhibitory cell types in shaping OD biases.

\section{HOW DOES LONG-TERM (14-DAY) MD SHIFT OD IN EXCITATORY AND INHIBITORY CELLS?}

To determine the effect of prolonged MD, Yazaki-Sugiyama et al. extended the period of deprivation to 14 days (LTMD). In pyramidal neurons LTMD did not produce any further change in OD, largely consistent with prior results (Gordon and Stryker, 1996; Frenkel and Bear, 2004). However, the FS cells again yielded surprises. LTMD led to responses dominated by the ipsilateral non-deprived eye, a reversal of the contralateral bias seen after 3 days MD. Thus Yazaki-Sugiyama et al. describe a bidirectional response to $\mathrm{MD}$ in FS cells: initially binocular neurons acquire contralateral (deprived) eye bias during the early phases of $\mathrm{MD}$, which reverses to ipsilateral (non-deprived) eye bias with longer periods of deprivation (Figure 1, top row).

A strong OD shift away from the deprived eye in FS neurons following prolonged MD is consistent with the observations of Gandhi et al. in the GAD67-GFP mice (Gandhi et al., 2008). In contrast, parvalbumin-positive cells failed to exhibit a shift in OD following 120 days of MD when assayed via visually evoked c-Fos in rats (Mainardi et al., 2009). These discrepant findings may be the result of varying plasticity among interneuron subtypes or speciesspecific mechanisms.

To investigate a possible mechanism behind the bidirectional response to $\mathrm{MD}$ in FS cells, the authors present modeling data showing that a spike-timing-dependent plasticity (STDP) rule applied to excitatory inputs onto inhibitory neurons, if properly constructed, predicts a bidirectional OD shift in accordance with the observed data. However, it is not clear whether this STDP rule has a physiological basis in layer 2/3 FS cells in mouse visual cortex. First, Yazaki-Sugiyama et al. show that the STDP rule is by itself insufficient to account for the eventual loss of deprived-eye responses and reversal of spike bias observed with prolonged MD because the rule favors elimination of active synapses over inactive ones. To compensate, the STDP rule must be supplemented by the slow elimination of inactive synapses. Second, the shape of the STDP rule used in the model is based on recordings from rat somatosensory cortex and includes regions in which synaptic weights are potentiated, which were not observed in the experimental data (Lu et al., 2007). These caveats aside, the modeling exercise shows that the observed changes are feasible using mechanisms of long-term potentiation and LTD of excitatory connections onto inhibitory neurons.

\section{DOES INHIBITORY MODULATION OF OCULAR BIAS CONTRIBUTE TO EXPRESSION OF THE OD SHIFT?}

The most robust and unexpected finding by Yazaki-Sugiyama et al. is the apparent reversal of spike bias (OD) when PTX is allowed to diffuse into pyramidal neurons following MD in juvenile animals (Figure 1). Interestingly, this same effect was not observed when adult ( $\sim$ P60) neurons were infused with PTX. Together, these findings imply that the effect of inhibition on expression of OD is developmentally transient.

Early work in mice suggested that MD also fails to shift OD when it is initiated after about 5 weeks of age (Gordon and Stryker, 1996), consistent with the well established concept of a critical period for visual cortical plasticity. However there is now broad agreement from multiple labs utilizing multiple methods of analysis that adult $(\sim \mathrm{P} 60)$ MD produces a robust OD shift in mouse visual cortex (Sawtell et al., 2003; Lickey et al., 2004; Pham et al., 2004; Tagawa et al., 2005; Frenkel et al., 2006; Hofer et al., 2006, 2009; Fischer et al., 2007; Sato and Stryker, 2008; Kameyama et al., 2010). Indeed, Yazaki-Sugiyama et al. also show that spike bias is shifted in adults after MD. In non-MD mice, neurons with ipsilaterally dominated responses were not observed, whereas MD progressively increased the percentage of cells dominated by the non-deprived 
eye such that after LTMD, 50\% of neurons exhibited an apparent OD shift and were now dominated by the non-deprived ipsilateral eye (Figure 2).

It is therefore puzzling that the authors appear to argue that the observed absence of changes in spike bias following PTX infusion in adults is evidence that inhibitory network changes are critical to OD plasticity. The repeated finding that OD plasticity is in fact induced by adult MD suggests instead the opposite conclusion: reorganization of inhibitory function is not a necessary component of OD shifts.

\section{TECHNICAL CONSIDERATIONS}

A novel aspect of the study by Yazaki-Sugiyama et al. is the use of intracellular PTX infusion through sharp microelectrodes to block GABA receptor conductances. To demonstrate the efficacy of intracellular PTX, the authors examined miniature inhibitory postsynaptic currents (mIPSCs) in slices of visual cortex using whole-cell patch recordings with internal solution containing PTX. While whole cell recordings make possible the resolution of mIPSCs using voltage clamp, they differ from sharp electrode recordings quite dramatically when it comes to the rate and extent of intracellular dialysis. Demonstrating the time-course for the elimination of IPSPs evoked through electrical stimulation or GABA iontophoresis by PTX delivered through sharp electrodes in slice would have better validated this approach in vivo.

A related concern is that the use of a cesium acetate based internal solution in the PTX experiments as opposed to a potassium acetate solution for other experiments in which PTX was not applied. Cesium is a well-known blocker of a variety of potassium channels, and as such will have effects on dendritic cable properties, membrane potential, and spike output, in addition to eliminating the effects of $\mathrm{GABA}_{\mathrm{B}}$ receptors. This may account for the apparent low value of contralateral bias in non-MD animals prior to PTX infusion (average bias approximately 0.13 ) when compared to the average bias reported from experiments using potassium acetate filled electrodes (average bias approximately 0.3 ).

Lastly, it must be noted that the use of barbiturate anesthesia by Yazaki-Sugiyama et al. could potentially confound the results by binding $\mathrm{GABA}_{\mathrm{A}}$ receptors and accentuating inhibition within the cortex. Previous work has shown that administration of barbiturates can in some circumstances alter OD (Pham et al., 2004). In contrast, the in vivo calcium imaging studies discussed here used isoflurane (Gandhi et al., 2008) or urethane (Kameyama et al., 2010), whereas anesthetic was absent during c-Fos induction experiments (Mainardi et al., 2009). The choice of anesthetic used while measuring OD may therefore contribute in part to the contrasting reports of OD plasticity in inhibitory cells.

\section{CONCLUDING REMARKS}

There is considerable evidence linking the developmental regulation of cortical plasticity to the maturation of inhibition, however the contribution of inhibitory neurons to OD plasticity has been more difficult to determine. The recent studies discussed in this review have clearly demonstrated that inhibition is affected by MD and may play a role in shaping the physiological response to sensory deprivation. However, the relative significance of inhibitory plasticity in maintaining an OD shift after MD remains uncertain,

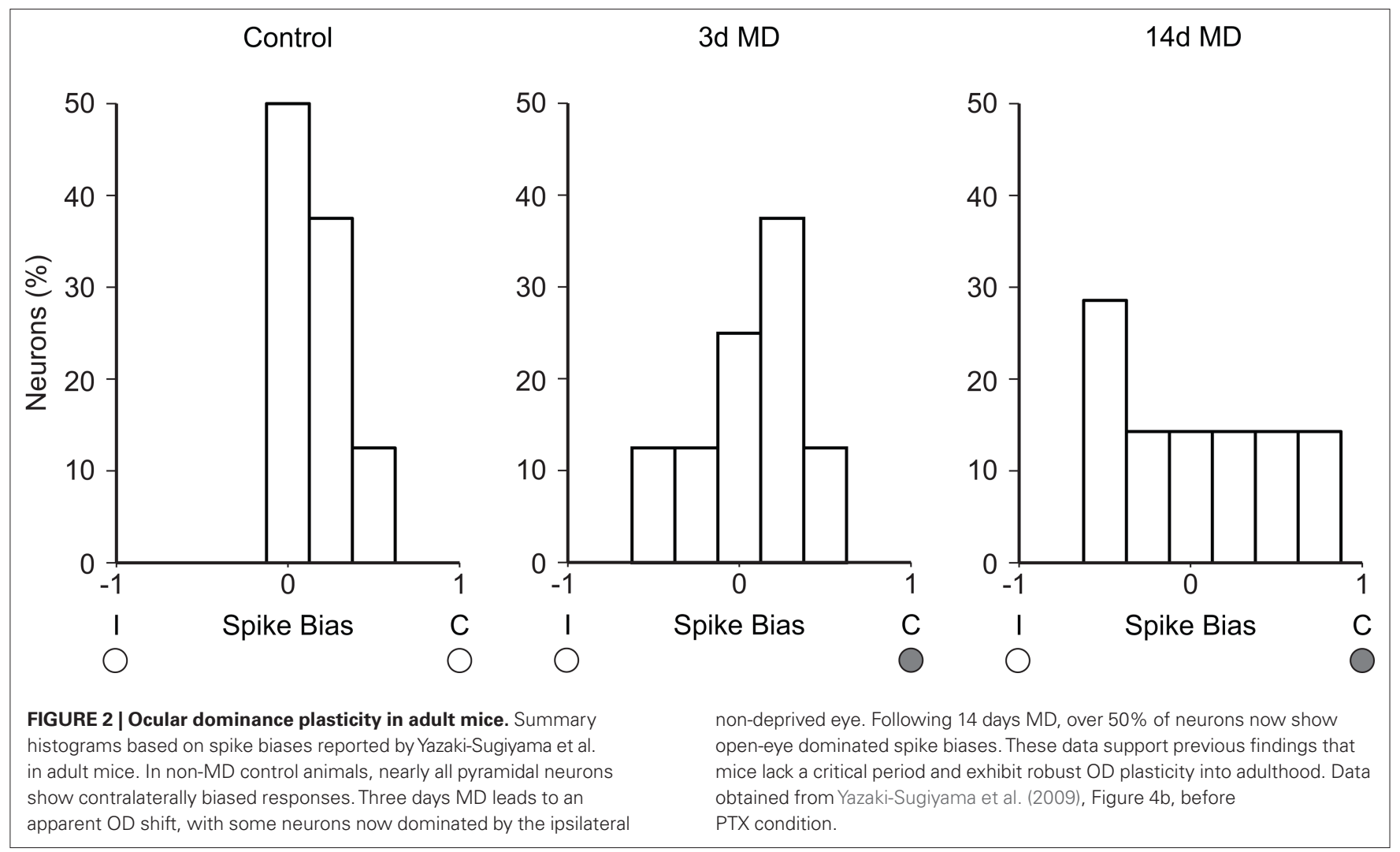


particularly in light of the recent finding that expression of an OD shift can be accounted for solely through changes in the strength of excitatory thalamocortical transmission (Khibnik et al., 2010).

The results presented by Yazaki-Sugiyama et al. provide novel insights into the effects of MD on inhibition and raise several interesting questions about the role of inhibitory neurons in OD that need to be addressed with future experiments. The initial binocularity of FS neurons and their paradoxical shift toward the deprived eye have not been observed previously (e.g., Gandhi et al., 2008; Mainardi et al., 2009; Kameyama et al., 2010). Recent advances in extracellular recording techniques facilitate the distinction between excitatory and inhibitory neurons in mice (Niell and Stryker, 2008). Applying this approach to OD plasticity would allow both confirmation of the paradoxical shift in FS neurons and the extension of these results to deeper cortical layers. Additionally, a recently generated parvalbumin-Cre mouse (Hippenmeyer et al., 2005) can be used to genetically label parvalbumin-positive neurons for in vivo calcium imaging experiments, thereby restricting analysis to a particular class of inhibitory cells while avoiding the confound of reduced GABA levels in GAD67-GFP knock-in mice (Tamamaki et al., 2003; Gandhi et al., 2008). Similar experiments examining OD plasticity in other classes of genetically identified interneurons could determine the source of monocularly biased inhibition required to explain the loss of pyramidal cell bias following

\section{REFERENCES}

Burchfiel, J. L., and Duffy, F. H. (1981). Role of intracortical inhibition in deprivation amblyopia: reversal by microiontophoretic bicuculline. Brain Res. 206, 479-484.

Coleman, J. E., Law, K., and Bear, M. F. (2009). Anatomical origins of ocular dominance in mouse primary visual cortex. Neuroscience 161, 561-571.

Crozier, R. A., Wang, Y., Liu, C. H., and Bear, M. F. (2007). Deprivationinduced synaptic depression by distinct mechanisms in different layers of mouse visual cortex. Proc. Natl. Acad. Sci. U.S.A. 104, 1383-1388.

Duffy, F. H., Burchfiel, J. L., and Conway, J. L. (1976). Bicuculline reversal of deprivation amblyopia in the cat. Nature 260, 256-257.

Fischer, Q. S., Graves, A., Evans, S., Lickey, M. E., and Pham, T. A. (2007). Monocular deprivation in adult mice alters visual acuity and single-unit activity. Learn. Mem. 14, 277-286.

Frenkel, M.Y., and Bear, M. F. (2004). How monocular deprivation shifts ocular dominance in visual cortex of young mice. Neuron 44, 917-923.

Frenkel, M. Y., Sawtell, N. B., Diogo, A. C., Yoon, B., Neve, R. L., and Bear, M. F. (2006). Instructive effect of visual experience in mouse visual cortex. Neuron 51, 339-349.

Gandhi, S. P., Yanagawa, Y., and Stryker, M. P. (2008). Delayed plasticity of inhibitory neurons in developing visual

delivery of PTX. Lastly, the discovery of genetic means to transiently silence populations of neurons (e.g., allatostatin receptors or halorhodopsin; Lechner et al., 2002; Zhang et al., 2007) combined with advanced recording techniques (Niell and Stryker, 2008) suggests that it is ultimately possible to determine the contribution of inhibitory inputs from specified cell types to the response properties of both excitatory and inhibitory neurons across cortical layers.

Applying Occam's razor - the notion that the simplest solution is usually the correct one - to the problem of OD plasticity has always suggested that the initial contralateral bias and its subsequent loss after MD could both be well explained by the relative strength and plasticity of eye-specific excitatory inputs to visual cortex. The results of Yazaki-Sugiyama et al. suggest the possibility that the maintenance and modification of OD in visual cortex may be far more complex than assumed previously. Although there is no reason to discard the well-supported and simple model of OD plasticity via modifications of thalamocortical input, these and other recent studies suggest ways it might be amended in the future to include inhibitory influences.

\section{ACKNOWLEDGMENTS}

We thank Arnold Heynen and Lena Khibnik for helpful discussions, and Suzanne Meagher and Erik Sklar for administrative and technical support.

excitatory cortical neurons. J. Neurosci. 30, 1551-1559.

Katagiri, H., Fagiolini, M., and Hensch, T. K. (2007). Optimization of somatic inhibition at critical period onset in mouse visual cortex. Neuron 53, 805-812.

Khibnik, L., Cho, K. K., and Bear, M. F. (2010). Relative contribution of feedforward excitatory connections to expression of ocular dominance plasticity in layer 4 of visual cortex. Neuron 66, 493-500.

Lechner, H.A., Lein, E. S., and Callaway, E. M. (2002).Agenetic method for selective and quickly reversible silencing of mammalian neurons. J. Neurosci. 22, 5287-5290.

Lickey, M. E., Pham, T.A., and Gordon, B. (2004). Swept contrast visual evoked potentials and their plasticity following monocular deprivation in mice. Vision Res. 44, 3381-3387.

Lu, J. T., Li, C. Y., Zhao, J. P., Poo, M. M., and Zhang,X.H.(2007). Spike-timingdependent plasticity of neocortical excitatory synapses on inhibitory interneurons depends on target cell type. J. Neurosci. 27, 9711-9720.

Maffei, A., Nataraj, K., Nelson, S. B., and Turrigiano, G. G. (2006). Potentiation of cortical inhibition by visual deprivation. Nature 443, 81-84.

Mainardi, M., Landi, S., Berardi, N., Maffei, L., and Pizzorusso, T. (2009). Reduced responsiveness to longterm monocular deprivation of par- valbumin neurons assessed by c-Fos staining in rat visual cortex. PLoS ONE 4, e4342. doi: 10.1371/journal. pone.0004342.

McCurry, C. L., Shepherd, J. D., Tropea, D., Wang, K. H., Bear, M., and Sur, M. (2010). Loss of Arc renders the visual corteximpervious to the effects of sensory experience or deprivation. Nat. Neurosci. 13, 450-457.

Mitzdorf, U., and Singer, W. (1980). Monocular activation of visual cortex in normal and monocularly deprived cats: an analysis of evoked potentials. J. Physiol. 304, 203-220.

Nelson, S., Toth, L., Sheth, B., and Sur, M. (1994). Orientation selectivity of cortical neurons during intracellular blockade of inhibition. Science 265, 774-777.

Niell, C. M., and Stryker, M. P. (2008). Highly selective receptive fields in mouse visual cortex. J. Neurosci. 28 , 7520-7536.

Pham, T. A., Graham, S. J., Suzuki, S., Barco,A., Kandel, E. R., Gordon, B., and Lickey, M.E. (2004). A semi-persistent adult ocular dominance plasticity in visual cortex is stabilized by activated CREB. Learn. Mem. 11, 738-747.

Sato, M., and Stryker, M. P. (2008). Distinctive features of adult ocular dominance plasticity. J. Neurosci. 28, 10278-10286.

Sawtell, N. B., Frenkel, M. Y., Philpot, B. D., Nakazawa, K., Tonegawa, S., and Bear, M. F. (2003). NMDA 
receptor-dependent ocular dominance plasticity in adult visual cortex. Neuron 38, 977-985.

Shatz, C. J., and Stryker, M. P. (1978). Ocular dominance in layer IV of the cat's visual cortex and the effects of monocular deprivation. J. Physiol. 281, 267-283.

Sillito, A. M. (1975). The contribution of inhibitory mechanisms to the receptive field properties of neurones in the striate cortex of the cat. J. Physiol. 250, 305-329.

Sillito, A. M., Kemp, J.A., and Blakemore, C. (1981). The role of GABAergic inhibition in the cortical effects of monocular deprivation. Nature 291, 318-320.

Singer, W. (1977). Effects of monocular deprivation on excitatory and inhibitory pathways in cat striate cortex. Exp. Brain Res. 30, 25-41.

Smith, G. B., Heynen, A. J., and Bear, M. F. (2009). Bidirectional synaptic mechanisms of ocular dominance plasticity in visual cortex. Philos. Trans. $R$. Soc. Lond., B, Biol. Sci. 364, 357-367.

Swadlow, H. A. (1988). Efferent neurons and suspected interneurons in binocular visual cortex of the awake rabbit: receptive fields and binocular properties. J. Neurophysiol. 59, 1162-1187.

Tagawa, Y., Kanold, P. O., Majdan, M., and Shatz, C. J. (2005). Multiple periods of functional ocular dominance plasticity in mouse visual cortex. Nat. Neurosci. 8, 380-388.

Tamamaki, N., Yanagawa, Y., Tomioka, R., Miyazaki, J., Obata, K., and Kaneko, T. (2003). Green fluorescent protein expression and colocalization with calretinin, parvalbumin, and somatostatin in the GAD67-GFP knock-in mouse. J. Comp. Neurol. 467, 60-79.

Tsumoto, T., and Suda, K. (1978). Evidence for excitatory connections from the deprived eye to the visual cortex in monocularly deprived kittens. Brain Res. 153, 150-156.

Wiesel, T. N., and Hubel, D. H. (1963). Single-cell responses in striate cortex of kittens deprived of vision in one eye. J. Neurophysiol. 26, 1003-1017.

Yazaki-Sugiyama, Y., Kang, S., Cateau, H., Fukai, T., and Hensch, T. K. (2009). Bidirectional plasticity in fast-spiking GABA circuits by visual experience. Nature 462, 218-221.

Yoon, B. J., Smith, G. B., Heynen, A. J., Neve, R. L., and Bear, M. F. (2009). Essential role for a long-term depression mechanism in ocular dominance plasticity. Proc. Natl. Acad. Sci. U.S.A. 106, 9860-9865.

Zhang, F., Wang, L.P., Brauner, M., Liewald, J. F., Kay, K., Watzke, N., Wood, P. G., Bamberg, E., Nagel, G., Gottschalk, A., and Deisseroth, K. (2007). Multimodal fast optical interrogation of neural circuitry. Nature 446, 633-639.
Conflict of Interest Statement: The authors declare that the research was conducted in the absence of any commercial or financial relationships that could be construed as a potential conflict of interest.

Received: 22 March 2010; paper pending published: 12 May 2010; accepted: 28 May 2010; published online: 17 June 2010.

Citation: Smith GB and Bear MF (2010)

Bidirectional ocular dominance plasticity of inhibitory networks: recent advances and unresolved questions. Front. Cell. Neurosci. 4:21. doi: 10.3389/fncel.2010.00021

Copyright (C) 2010 Smith and Bear. This is an open-access article subject to an exclusive license agreement between the authors and the Frontiers Research Foundation, which permits unrestricted use, distribution, and reproduction in any medium, provided the original authors and source are credited. 